\title{
Laparoscopic and endoscopic cooperative surgery plus sentinel lymph node navigation surgery for elderly patients with early gastric cancer - phase II trial: Study protocol
}

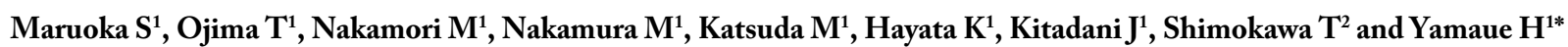 \\ ${ }^{1}$ Second Department of Surgery, School of Medicine, Wakayama Medical University, Wakayama, Japan \\ ${ }^{2}$ Clinical Research Center, School of Medicine, Wakayama Medical University, Wakayama, Japan
}

\begin{abstract}
Background: When patients are outside the criteria for endoscopic submucosal dissection (ESD) treatment, additional gastrectomy with radical lymph node dissection is generally performed. It is thought, however, that post-gastrectomy syndrome and decline in quality of life occur in elderly patients. In patients with submucosal-invasive gastric cancer who undergo gastrectomy, lymph node metastases (LNM) are very common. LNM in patients with submucosal-invasive gastric cancer after ESD, however, are very rare. In elderly patients who need additional treatment after ESD, partial gastrectomy with regional lymphadenectomy may be feasible in oncological. It is extremely difficult to determine the appropriate incision line in the laparoscopic view because of scarring in the intraluminal stomach after gastric ESD. We plan to perform laparoscopic and endoscopic cooperative surgery (LECS) to address this problem.

Methods: When performing LECS for patients with early gastric cancer (EGC), sufficient lymph node dissection is required, so we apply sentinel lymph node navigation surgery (SNNS). The purpose of this trial is to test the hypothesis that limited operation using LECS plus SNNS is effective and oncologically safe in the selected group including elderly patients who undergo ESD.

Conclusion:-This study is a prospective, single arm, single center trial. Patients over 75 years old with EGC who are pathologically diagnosed outside the criteria for ESD treatment are eligible. The primary endpoint is to determine the rate of the postoperative complications. Secondary endpoints include determination of surgical results, postoperative outcomes, and survival rates.
\end{abstract}

\begin{abstract}
Abbreviations: ESD: endoscopic submucosal dissection; LNM: lymph node metastasis; LECS: laparoscopic and endoscopic cooperative surgery; EGC: early gastric cancer; SNNS: sentinel lymph node navigation surgery; QOL: quality of life; ICG - indocyanine green; OS: overall survival; DSS: disease-specific survival; DFS: disease-free survival; SNs: sentinel lymph nodes; RCT: randomized controlled trial
\end{abstract}

\section{Introduction}

The number of patients with early gastric cancer (EGC) who undergo endoscopic treatment is increasing worldwide. Accurate diagnosis of invasive depth (clinical T factor) of gastric cancer is difficult [1], so in clinical practice, diagnostic endoscopic submucosal dissection (ESD) is often performed. If patients are outside criteria for ESD treatment, additional gastrectomy with radical lymph node dissection is generally performed [2]. It is generally accepted, however, that patients who undergo radical gastrectomy experience post-gastrectomy syndrome, such as appetite loss, epigastric discomfort, reflux symptom, weight loss, and decline in quality of life (QOL). Such post-gastrectomy syndromes appear remarkably in elderly patients $[3,4]$.

After gastrectomy with submucosal-invasive gastric cancer, lymph node metastases (LNM) are very common (10 to 23\%) [5-7]. LNM in patients after ESD are reportedly rare (0 to $5 \%$ ), and good prognosis in patients with EGC treated with ESD may be obtained [8-10]. The site of LNM was the perigastric lymph nodes in all patients who underwent
ESD [10]. Therefore, in elderly patients who need additional treatment after ESD, partial gastrectomy with regional lymphadenectomy may be oncologically feasible.

Scarring is present after gastric ESD in intraluminal stomach, so determining the appropriate incision line is extremely difficult by laparoscopic view. In recent years, laparoscopic and endoscopic cooperative surgery (LECS) was developed for gastric submucosal tumor [11] and the usefulness and feasibility were reported [12,13]. We therefore apply LECS to partial gastrectomy in patients with EGC after ESD and by minimizing the range of resection can improve postoperative QOL [14].

When performing LECS for patients with EGC, sufficient lymph node dissection is required to prevent recurrence of gastric cancer. If limited operation is adapted for EGC patients, increase in recurrence rate is not permissible, so lymph node dissection should be minimized by sentinel lymph node navigation surgery (SNNS). False negatives are a problem in conventional pigmentary visualization method [15]. Recently, however, there has been success in reducing false negative rate

*Correspondence to: Hiroki Yamaue, MD, Second Department of Surgery, School of Medicine, Wakayama Medical University, 811-1, Kimiidera, Wakayama 641-8510, Japan, E-mail: yamaue-h@wakayama-med.ac.jp

Key words: stomach neoplasms; Gastrectomy; Sentinel lymph node biopsy

Received: April 25, 2018; Accepted: April 28, 2018; Published: April 30, 2018 
Maruoka S (2018) Laparoscopic and endoscopic cooperative surgery plus sentinel lymph node navigation surgery for elderly patients with early gastric cancer phase II trial: Study protocol

by using new modalities and tracers [16-18] particularly the method combining indocyanine green (ICG) and infrared light observation, which reduced the false negative rate to $0 \%[19,20]$. The purpose of this trial is to test the hypothesis that limited operation using LECS plus SNNS (LECS-SNNS trial) is effective and oncologically safe in the selected group including elderly patients who undergo ESD.

LECS-SNNS trial is conducted in accordance with the Declaration of Helsinki. Wakayama Medical University Hospital Institutional Review Board approved the final version of the protocol (version 1.0) prior to the start of the study (approval number: 2235). The University Hospital Medical Information Network Clinical Trials Registry number is UMIN000031003 (https://upload.umin.ac.jp/cgi-open-bin/ctr_e/ctr_ view.cgi? recptno=R000035311)

\section{Materials and methods}

Objectives: This trial aims to test the hypothesis that limited operation using LECS plus SNNS is effective and oncologically safe in the selected group, specifically elderly patients with EGC who were diagnosed as being outside the criteria for ESD treatment pathologically.

Study setting: The LECS-SNNS trial is a prospective, single arm, single center trial.

End points: The primary endpoint is to ascertain the rate of postoperative complications, which are analyzed according to DindoClavien classification [21]. Complications higher than grade II are regarded as clinically significant. Secondary endpoints are: (i) surgical results, such as operation time, blood loss, and transition rate to open surgery; (ii) postoperative course, such as time to start of drinking, time to start of eating, postoperative hospital stay, weight loss rate, serum albumin, serum pre-albumin, and postoperative quality of life; (iii) oncological outcomes, such as overall survival (OS), disease specific survival (DSS), disease free survival (DFS). OS duration is defined as the time between operation and death. DSS duration is defined as the time from operation to death due to relapse. DFS duration is defined as the time from operation to relapse. QOL is assessed with FACT-G, FACT-Ga and FACT-Ga TOI [22-24].

Inclusion criteria: Patients over 75 years old with EGC selected to undergo additional resection after ESD are eligible for inclusion. Written informed consent is required.

\section{Exclusion criteria:}

1. History of preoperative chemotherapy for gastric cancer.

2. Ischemic heart disease requiring treatment.

3. Liver cirrhosis or active hepatitis

4. Severe respiratory disease (including interstitial pneumonia, pulmonary fibrosis, pulmonary emphysema).

5. Chronic renal failure requiring hemodialysis.

6. Other malignant disease that can influence adverse events.

7. Patients in whom laparoscopic surgery cannot be performed because of history of laparotomy in the upper abdomen.

Registration: After confirming fulfillment of the eligibility criteria, all handling cases are managed by subject identification code or anonymized registration number. The correspondence table of the anonymizing code and the name and the consent form containing the name are strictly kept in the separate lockable document storage of the Second Department of Surgery.

\section{Treatment methods}

SNNS: Under general epidural anesthesia, the patient is placed in the supine position with open legs and pneumoperitoneum of 10 $\mathrm{mmHg}$ is created. A laparoscope is inserted through the umbilical incision, and four trocars are placed in the upper abdomen. The overtube is inserted under the oral endoscope. We confirm the scar of endoscopic treatment and then a 0.5\% ICG solution (Diagnogreen $0.5 \%$; Daiichi Pharmaceutical, Tokyo, Japan) is endoscopically injected into the submucosa of the gastric wall at four sites $(0.5 \mathrm{~mL}$ each $)$ around the scar intraoperatively. After 20 minutes, ICG fluorescence imaging is carried out using the 1588 AIM camera system with Endoscopic Near - Infrared Visualization (Stryker Corporation, Kalamazoo, MI). Fluoresced lymph nodes are considered to be sentinel lymph nodes (SNs). SNs and surrounding sentinel lymphatic basin are extracted from the fatty tissue. Lymph nodes in the dissected specimens are isolated from the surrounding tissues on the back table. These lymph nodes are submitted for the intraoperative rapid medical examination (Figure 1).

LECS: Details of the LECS procedures performed at our institute have been previously described [12]. Blood vessels and omentum in the excision area around the scar are prepared using an ultrasonically activated scalpel (HARMONIC; Ethicon Endo-Surgery, Inc., Cincinnati, OH). The jejunum near the ligament of Treitz is clamped using detachable forceps to avoid air inflation in the gastrointestinal tract. With a view of the endoscopic image, a circumferential incision is made around the scar using an ESD technique involving a Flush Knife (Fujinon Optical Co., Ltd., Tokyo, Japan), which is used to perforate the stomach. The IT knife (Olympus, Tokyo, Japan) tip is inserted into the perforation site and seromuscular dissection is initiated along the incision line of the submucosal layer. With a view of the laparoscopic image, the seromuscular layer of the stomach is dissected along the incision line using HARMONIC, and the scar is removed. The postexcisional hole in the stomach is closed using a laparoscopic suturing technique.

If intraoperative pathologic diagnosis is metastatic lymph nodes, radical gastrectomy with D2 lymphadenectomy is performed (Figure 1).

Postoperative management: Nasogastric tube will be removed when awoken from anesthesia. Postoperative pain control consists of patient-controlled analgesia. Patients are encouraged to be out of bed and walking around the ward under the guidance of a physiotherapist or nurse the day after surgery. Patients will be discharged when they pass stool, are able to drink, can walk and are comfortable with oral analgesia.

Follow-up: All registered patients will be followed up for at least 5 years. Blood test, upper gastrointestinal endoscope, and enhanced abdominal computed tomography will be evaluated at least 5 years. Assessment of QOL will be performed preoperatively, at discharge, after six months, one year, two years, three years, four years, and five years postoperatively (Table 1).

Study design and statistical analysis: The planned sample size of the phase II study is 20 patients. Primary endpoint of LECS-SNNS trial is to determine the rate of postoperative complications. In the main analysis, the point estimate and the $95 \%$ confidence interval are constructed for the rate of postoperative complications. Total postoperative complications in radical gastrectomy in our department so far are $10 \%[4,25]$. If the lower limit of the $95 \%$ confidence 
Maruoka S (2018) Laparoscopic and endoscopic cooperative surgery plus sentinel lymph node navigation surgery for elderly patients with early gastric cancer phase II trial: Study protocol

- Over 75 years-old

\section{Eligibility}

- Gastric cancer requiring additional resection after *ESD All non-applied lesions or Non-curative resection

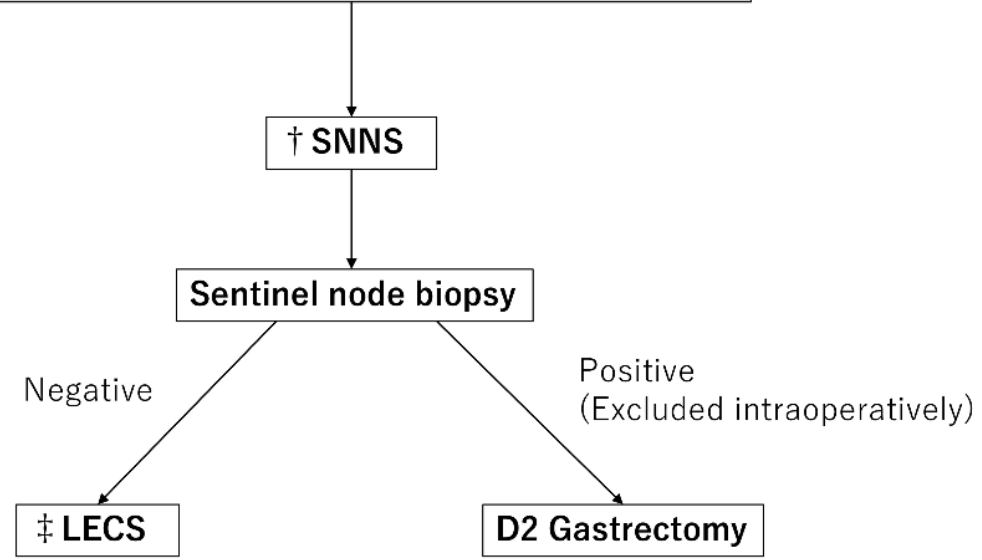

Figure 1: Treatment design of LECS-SNNS trial.

$*$ ESD $=$ Endoscopic submucosal dissection. $†$ SNNS $=$ Sentinel lymph node navigation surgery, $\$$ LECS $=$ Laparoscopic and endoscopic cooperative surgery

Table 1: Data collection at follow-up assessment.

\begin{tabular}{|c|c|c|c|c|c|c|c|c|c|}
\hline \multirow[b]{2}{*}{ TIMEPOINT } & \multirow{2}{*}{$\begin{array}{c}\text { Enrolment } \\
-28 \text { days }\end{array}$} & \multirow{2}{*}{\begin{tabular}{|c|} 
Day of surgery \\
$0 \sim 14$ days \\
\end{tabular}} & \multirow{2}{*}{\begin{tabular}{|c|} 
Discharge Date \\
$0 \sim 14$ days \\
\end{tabular}} & \multicolumn{6}{|c|}{ Postoperative Months } \\
\hline & & & & 6 & 12 & 24 & 36 & 48 & 60 \\
\hline Informed Consent & $\mathrm{X}$ & & & & & & & & \\
\hline Blood Test & $\mathrm{X}$ & & & $\mathrm{X}$ & $\mathrm{X}$ & $\mathrm{X}$ & $\mathrm{X}$ & $\mathrm{X}$ & $\mathrm{X}$ \\
\hline Endoscope & $\mathrm{X}$ & & & $\mathrm{X}$ & $\mathrm{X}$ & $\mathrm{X}$ & $\mathrm{X}$ & $\mathrm{X}$ & $\mathrm{X}$ \\
\hline *CT Scan & $\mathrm{X}$ & & & $\mathrm{X}$ & $\mathrm{X}$ & $\mathrm{X}$ & $\mathrm{X}$ & $\mathrm{X}$ & $\mathrm{X}$ \\
\hline Surgical Record & & $\mathrm{X}$ & & & & & & & \\
\hline Postoperative Record & & & $\mathrm{X}$ & & & & & & \\
\hline Pathological Findings & & & $\mathrm{X}$ & & & & & & \\
\hline Weight & & & & $\mathrm{X}$ & $\mathrm{X}$ & $\mathrm{X}$ & $\mathrm{X}$ & $\mathrm{X}$ & $\mathrm{X}$ \\
\hline †QOL Survey & $\mathrm{X}$ & & $\mathrm{X}$ & $\mathrm{X}$ & $\mathrm{X}$ & $\mathrm{X}$ & $\mathrm{X}$ & $\mathrm{X}$ & $\mathrm{X}$ \\
\hline
\end{tabular}

${ }^{*} \mathrm{CT}$ scan $=$ computed tomography scan. $\dagger \mathrm{QOL}=$ quality of life

interval exceeds $10 \%$, LECS and SNNS is judged to be an unacceptable technique. Data will be collected prospectively for all patients including history, physical examination, laboratory data, pathologic examination, perioperative clinical information and complications. Data is collected via datasheets on paper and kept securely. All required parameters will be collected in a SPSS data file (SPSS version 25, IBM statistics Chicago, IL).

\section{Discussion}

We planned LECS-SNNS trial for patients over 75 years old with EGC selected to undergo additional resection after ESD. This LECSSNNS trial is the first report of limited operation for elderly patients with EGC. If favorable outcomes are obtained in this study, this limited operation of LECS-SNNS may become a treatment option for elderly patients with EGC.

This trial has several limitations. It is a phase II study without randomized controlled trial (RCT) and is conducted at a single institution. Due to the small sample size, findings from this trial do not allow established clinical application, but rather serve to inform the need for larger multicenter phase III RCT.

\section{Competing interests}

No authors have competing interests to report.

\section{Authors' contributions}

Maruoka designed the protocol and wrote the manual. Ojima further aided in assessment and revision of the protocol and revised the manuscript. All authors approved the final version of the protocol.

\section{References}

1. Pei Q, Wang L, Pan J, Ling T, Lv Y, et al. (2015) Endoscopic ultrasonography for staging depth of invasion in early gastric cancer: A meta-analysis. $J$ Gastroenterol Hepatol 30:1566-73

2. Japanese Gastric Cancer Association. Japanese gastric cancer treatment guidelines (2014) (ver. 4) (2017). Gastric Cancer 20:1-19

3. Nakamura M, Yamaue H (2016) Reconstruction after proximal gastrectomy for gastric cancer in the upper third of the stomach: a review of the literature published from 2000 to 2014. Surg Today 46: 517-527

4. Nakamura M, Nakamori M, Ojima T, et al. (2016) Randomized clinical trial comparing long-term quality of life for Billroth I versus Roux-en-Y reconstruction after distal gastrectomy for gastric cancer. BJS 103: 337-347

5. An JY, Baik YH, Choi MG, Noh JH, Shon TS, et al. (2007) Predictive factors for lymph node metastasis in early gastric cancer with submucosal invasion. Ann Surg 246: 749753

6. Park YD, Chung YJ, Chung HY, et al. (2008) Factors related to lymph node metastasis and the feasibility of endoscopic mucosal resection for treating poorly differentiated adenocarcinoma of the stomach. Endoscopy 40: 7-10 
Maruoka S (2018) Laparoscopic and endoscopic cooperative surgery plus sentinel lymph node navigation surgery for elderly patients with early gastric cancer phase II trial: Study protocol

7. Nonaka S, Oda I, Nakaya T, et al. (2011) Clinical impact of a strategy involving endoscopic submucosal dissection for early gastric cancer: determining the optimal pathway. Gastric Cancer 14: 56-62

8. Choi IJ, Lee JH, Kim YI, et al. (2015) Long-term outcome comparison of endoscopic resection and surgery in early gastric cancer meeting the absolute indication for endoscopic resection. Gastrointestinal Endoscopy 81: 333-341

9. Ryu SJ, Kim BW, Kim BG, et al. (2016) Endoscopic submucosal dissection versus surgical resection for early gastric cancer: a retrospective multicenter study on immediate and long-term outcome over 5 years. Surg Endosc 30: 5283-5289

10. Ojima T, Takifuji K, Nakamura M, Nakamori M, Yamaue H, et al. (2016) Feasibility of Endoscopic Submucosal Dissection for Submucosal - invasive Gastric Cancer and the Predictors of Residual or Recurrent Cancer. Surg Laparosc Endosc Percutan Tech 26: 401-405

11. Hiki N, Yamamoto Y, Fukunaga T, et al (2008) Laparoscopic and endoscopic cooperative surgery for gastrointestinal stromal tumor dissection. Surg Endosc 22: $1729-1735$

12. Ojima T, Nakamura M, Nakamori M, et al. (2018) Laparoscopic and endoscopic cooperative surgery is a feasible treatment procedure for intraluminal gastric gastrointestinal stromal tumors compared to endoscopic intragastric surgery. Surg Endosc 32: 351-357

13. Ojima T, Nakamori M, Nakamura M, et al. (2018) Laparoscopic and Endoscopic Cooperative Surgery Versus Endoscopic Submucosal Dissection for the Treatment of Low-Risk Tumors of the Duodenum. J Gastrointest Surg 22: 935-940

14. Nomura E, Lee SW, Tokuhara T, Nitta T, Kawai M, et al. (2013) Functional Outcomes According to the Size of the Gastric Remnant and the Type of Reconstruction Following Distal Gastrectomy for Gastric Cancer: An Investigation Including Total Gastrectomy. Jpn J Clin Oncol 43: 1195-1202

15. Miyashiro I, Hiratsuka M, Sasako M, et al. (2014) High false-negative proportion of intraoperative histological examination as a serious problem for clinical application of sentinel node biopsy for early gastric cancer: final results of the Japan Clinical Oncology Group multicenter trial JCOG0302. Gastric Cancer 17: 316-323
16. Kitagawa Y, Takeuchi H, Takagi Y, et al. (2013) Sentinel Node Mapping for Gastric Cancer: A Prospective Multicenter Trial in Japan. J Clin Oncol 31: 3704-3710

17. Lee CM, Park S, Park SH, et al. (2017) Sentinel Node Mapping Using a Fluorescent Dye and Visible Light During Laparoscopic Gastrectomy for Early Gastric Cancer. Ann Surg 265: 766-773

18. Ichikura T, Sugasawa H, Sakamoto N, Yaguchi Y, Tsujimoto H, et al. (2009) Limited Gastrectomy with Dissection of Sentinel Node Stations for Early Gastric Cancer With Negative Sentinel Node Biopsy. Ann Surg 249: 942-947

19. Nimura H, Narimiya N, Mitsumori N, Yamazaki Y, Yanaga K, et al. (2014) Infrared ray electronic endoscopy combined with indocyanine green injection for detection of sentinel nodes of patients with gastric cancer. BJS 91: 575-579

20. Tajima Y, Yamazaki K, Masuda Y, et al. (2009) Sentinel Node Mapping Guided by Indocyanine Green Fluorescence Imaging in Gastric Cancer. Ann Surg 249: 58-62

21. Dindo D, Demartines N, Clavien PA (2004) Classification of surgical complications; a new proposal with evaluation in a cohort of 6336 patients and results of a survey. Ann Surg 240: 205-213

22. S. L. Eremenco (2004) FACT-Gastric: A new international measure of QOL in gastric cancer. J Clin Oncol 22: 755

23. Garland SN, Pelletier G, Lawe A, et al. (2011) Prospective Evaluation of the Reliability Validity, and Minimally Important Difference of the Functional Assessment of Cancer Therapy-Gastric(FACT-Ga) Quality-of-Life Instrument. Cancer 117:1302-12

24. Kawai M, Tani M, HironoS, et al. (2011) Pylorus ring resection reduces delayed gastric emptying in patients undergoing pancreatoduodenectomy: a prospective, randomized, controlled trial of pylorus-resecting versus pylorus-preserving pancreatoduodenectomy. Ann Surg 253: 495-501

25. Nakamura M, Nakamori M, Ojima T, et al. (2014) Reconstruction after proxima gastrectomy for early gastric cancer in the upper third of the stomach: An analysis of our 13-year experience. Surgery 156: 57-63.

Copyright: (C2018 Maruoka S. This is an open-access article distributed under the terms of the Creative Commons Attribution License, which permits unrestricted use, distribution, and reproduction in any medium, provided the original author and source are credited. 